\title{
The application of plasticity principles to friction
}

C M Churchman, A M Korsunsky, and D A Hills*

Department of Engineering Science, University of Oxford, Oxford, UK

The manuscript was received on 30 November 2004 and was accepted after revision for publication on 2 March 2006.

DOI: 10.1243/03093247JSA96

\begin{abstract}
This paper presents a compelling analogy between the ideas of plastic shakedown and frictional shakedown. It introduces the frictional equivalent of the Bree diagram and classifies the different possible responses of a simple system to typical periodic or cyclic loading.
\end{abstract}

Keywords: plastic shakedown, frictional shakedown, plasticity principles, friction, contacts

\section{INTRODUCTION}

The concept of 'shakedown' in structures made from elastoplastic material is well known and has been studied for half a century or more. This is just one form of the response that a structure subject to severe cyclic loading can display, others being a permanently elastic response and steady state plasticity. The last may conveniently be divided into behaviour in which the plastic strains imparted in each cycle accumulate, which is known as incremental collapse or ratcheting, and a response in which the plastic strains experienced are the same after many cycles as after the first (or an early cycle). This is known as cyclic plasticity, or occasionally plastic shakedown. The loads on the structure may be in the form of external mechanical forces or from thermal loads, and a well-known way of displaying the response of the structure is on what has become known as a Bree diagram, after the pioneering work of Bree [1] in deducing what might now be thought of as a 'map' of the structural response.

Frictional interfacial contact has many similarities with plastic response, if frictional slip is thought of as being analogous to plastic slip, or yielding. Of course, plasticity and hence classical plastic shakedown may occur simultaneously, but, if this possibility is excluded by demanding that the absolute loads are low relative to the yield strength of the material, frictional shakedown may be investigated in isolation.

* Corresponding author: Department of Engineering Science, University of Oxford, Parks Road, Oxford OX1 3PJ, UK. email: david.hills@eng.ox.ac.uk
The parallel between plasticity and friction is quite strong: firstly, the highest load that may be sustained without plasticity is analogous to the highest forces that may be sustained in which all contacts within the structure remain adhered - the elastic limit. Secondly, structures that are initially plastic but which, after a finite number of cycles, settle to a completely elastic state, are said to lie within the shakedown limit. Similarly, structures where one or more of the contacts present is initially slipping, but which settle down to an adhered state, may also be thought of as having shaken down. In addition, structures that exceed the shakedown limit exhibit steady state plasticity or, here, steady state slip. Those that ratchet show, in a plasticity problem, accumulating deformation, usually leading to plastic collapse - in a frictional problem the slip displacement in each cycle accumulates, leading to a rigid body motion. Lastly, those structures that exhibit cyclic plasticity are ones that, in a continuum sense, show steady state plasticity and in a frictional contact problem show reversing slip, but with no net displacement.

The object of the present paper is to pursue a very simple contact problem that is the frictional equivalent of the Bree analysis. In the spirit of the Bree analysis all of the details of real problems of the class are omitted and the problem is reduced to its bare essentials so that the physical response can emerge. It is worth remarking that, to the authors' knowledge, no frictional equivalent of the classical shakedown theorems of Melan [2] and Koiter [3] have been proved for frictional problems, and, although 
there is a strong analogy between the normality condition in plastic flow and that relevant in slipping friction, a strict proof of the applicability of the plasticity theorems to frictional contacts is yet to be achieved. Although all real contact problems are of finite extent, and may therefore themselves show a number of responses (adhesion, partial slip, and sliding), here it is assumed that the contacting body has two discrete point contacts, which may be adhered or sliding. The general configuration is shown in Fig. 1(a) and the simplified geometry to be studied is shown as a freebody diagram in Fig. 1(b). The geometry is simply a rigid 'bridge' [shown in Fig. 1(a)] journalled between rollers to prevent all transverse movement, so that the force $Q$ becomes an output quantity, corresponding to the reaction force provided by the rollers and equal to $F_{2}-F_{1}$. The rigid bridge contacts an elastic bar at the points $A$ and B. Each of the contacts supports a normal load $P$ and a shear load $T$. If $|T|<f P$, where $f$ is the coefficient of friction, the contact is adhered. If this condition does not hold, it is sliding. The stiffness of the bar between the two pairs of feet is $k$ [Fig. 1(b)]
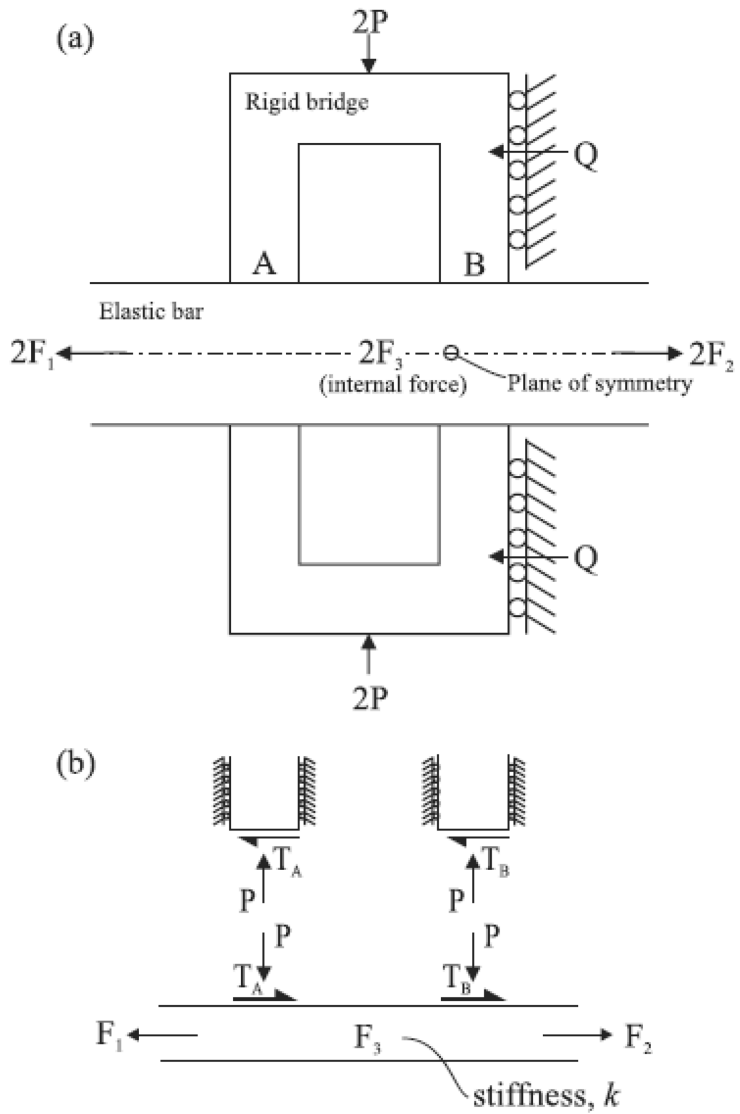

Fig. 1 Geometry of bridge contact: (a) full geometry, with the mirror image shown; (b) freebody diagram of a simplified contact geometry and various histories of the applied loads $F_{1}(t), F_{2}(t)$ are to be studied. The internal variable $F_{3}(t)$, which constitutes a representation of internal residual stress, is introduced as the force present in the bar between the two contacts.

It will be found that this extremely simple problem is sufficiently complex to show all the regimes cited above under certain combinations of the applied forces $F_{1}(t), F_{2}(t)$. Some tools must be introduced in order to depict clearly the different regimes. The first of these is analogous to a yield surface in plasticity and will be denoted a 'slip surface'. The slip surface for $F_{3}=0$ (i.e. no tension between the contacts) is shown in Fig. 2(a). Take a trajectory in $F_{1}, F_{2}$ space starting from the origin: when the slip surface is reached, the subsequent behaviour depends on which side or indeed corner of the square the trajectory hits. A trivial example is when $F_{2}=0$ is taken and $F_{1}$ is simply increased from 0 to some value $F_{1}^{\mathrm{MAX}}>f P$. This implies a vertical trajectory on Fig. 2(a) from the origin before hitting the line $F_{1} /(f P)=1$, from which point contact $A$ slips to the right relative to the bar and contact $B$ remains adhered, i.e. $\vec{A} \hat{B}$ in the present notation. This notation was devised to permit a succinct description of behaviour: $\hat{A}$ means that point $\mathrm{A}$ is adhered, $\vec{A}$ means that it is slipping to the right, and $\overleftarrow{A}$ that it is slipping to the left, as summarized in Fig. 2(b). It should be emphasized that, although A slips to the right relative to a corresponding point on the bar, in fact the bridge is assumed to be rigid, and so there can be no movement of contact $A$ relative to contact $B$. Therefore, when $F_{1}$ is increased beyond $f P$ the bar [in Fig. 1(b)]

(a)

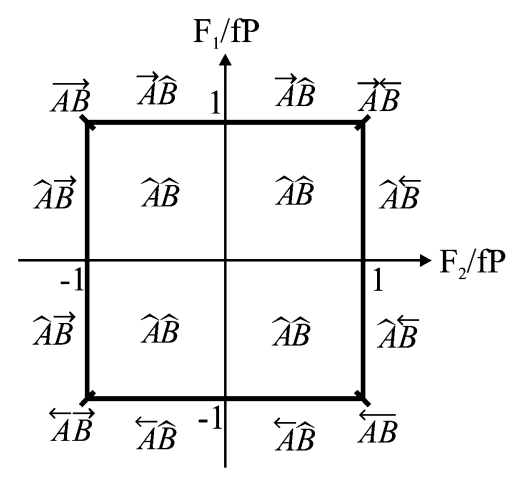

(b)

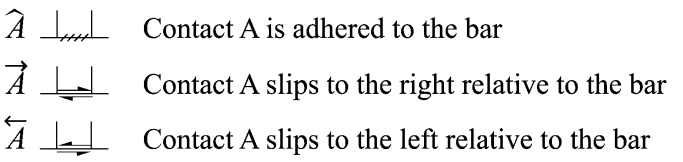

Fig. 2 (a) Slip surface for $F_{3} /(f P)=0$, showing the slip tendencies for each boundary; (b) notation employed 
(a)
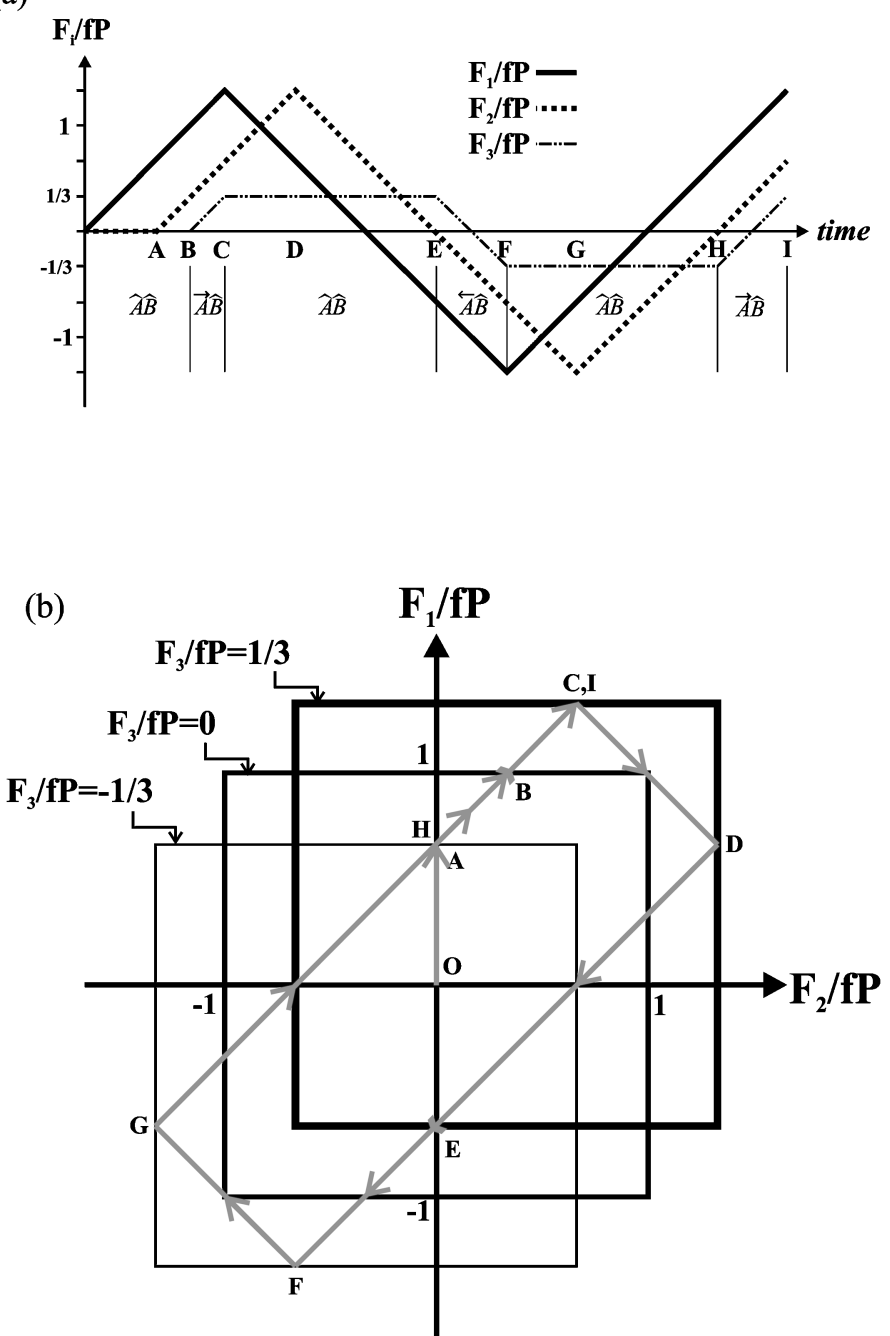

Fig. 3 (a) Example of triangular forcing functions where $F_{2}$ lags $F_{1}$ by $\pi / 4$; (b) trajectory depicted in $F_{1}-F_{2}$ space, showing the process of cyclic slip, $\vec{A} \hat{B} \Rightarrow \overleftrightarrow{A} \hat{B}$

stretches by $\Delta L$, which is proportional to the internal force $F_{3}, \Delta L=F_{3} / k$. As $F_{1}$ is increased further, $F_{3}$ takes up the extra tension that cannot be provided by the slipping contact $A$, i.e. $F_{3}=F_{1}-f P$. This then develops a differential force across contact $B$, and, because $F_{2}=0$, when $F_{1}$ reaches $2 f P$, there is gross sliding of both contacts $A$ and $B, \overrightarrow{A B}$.*

The problem just described provides an introductory example with monotonically increasing loading, going from both contacts adhered (cf. elastic problem), one foot slipping (cf. elastic-plastic problem) through to both contacts slipping (cf. a limit state).

\footnotetext{
* Because of the symmetry of the problem, a similar set of conditions would be observed when increasing $F_{2}$ while $F_{1}$ remained at zero.
}

\section{CYCLIC LOADING: EXAMPLE RESPONSE}

Figure 3(a) shows $F_{1}(t)$ and $F_{2}(t)$ as triangular waves of maximum $4 \mathrm{fP} / 3$ that are $\pi / 4$ out of phase, with $F_{2}$ lagging behind $F_{1}$. In Fig. 3(b) this temporal variation in the forcing functions $F_{1}(t)$ and $F_{2}(t)$ is shown as a trajectory in $F_{1}, F_{2}$ space, with salient points in the cycle marked with letters, A, ..., I. This diagram merits explanation. As the bar between the contacts is initially unloaded, $F_{3}=0$, and thus the operation takes place within the slip surface defined by the square centred on the origin of the coordinate set and delimited by the lines $F_{1}, F_{2}= \pm f P$ [the medium thickness line in Fig. 3(b)]. As $F_{1}, F_{2}$ increase in the manner shown $(\mathrm{O}, \mathrm{A}, \mathrm{B})$, point $\mathrm{B}$ is reached, which represents incipient slip for contact $A, \vec{A} \hat{B}$. In further increasing $F_{1}$ to its 
maximum $4 f P / 3(\mathrm{~B}, \mathrm{C})$, the dependent parameter $F_{3}$ increases to $f P / 3$. When $F_{1}$ starts to decrease $(C, D)$, the operation is now in a new square slip surface centred at $\left[F_{1} /(f P), F_{2} /(f P)\right]=(1 / 3,1 / 3)$ in the twodimensional figure [the thick line in Fig. 3(b) ]. Hence, point $\mathrm{D}$ is still within this slip surface and so $\hat{A} \overleftarrow{B}$ type behaviour is not seen. Instead, both contacts remain adhered $(\hat{A} \hat{B})$ from point $\mathrm{C}$ until point $\mathrm{E}$ is reached, where again the boundary of the slip surface is hit. Therefore, from $\mathrm{E}$ to $\mathrm{F}$ there is $\overleftarrow{A} \hat{B}$ type behaviour and the operating slip surface is 'pushed' to the $F_{3} /(f P)=-1 / 3$ square [the thinnest line in Fig. 3(b) ]. The path F, G, H is then fully adhered again, $\hat{A} \hat{B}$, until the segment $\mathrm{H}$, I is reached, which will result in $\vec{A} \hat{B}$ behaviour again. Thus, in the steady state the cyclic slip $\vec{A} \hat{B} \Leftrightarrow \overleftarrow{A} \hat{B}$ will be observed.

Note that other slip regimes may be found by simply changing the lag between $F_{1}$ and $F_{2}$. For example:

(a) $F_{2}$ lags $F_{1}$ by $\pi / 4$ (as before): $\vec{A} \hat{B} \Leftrightarrow \overleftarrow{A} \hat{B}$

(b) $F_{1}$ lags $F_{2}$ by $\pi / 4: \hat{A} \overleftarrow{B} \Leftrightarrow \hat{A} \vec{B}$

(c) $F_{1}$ in phase with $F_{1}: \vec{A} \overleftarrow{B} \Leftrightarrow \overleftarrow{A} \vec{B}$

(d) $F_{1}$ in antiphase with $F_{2}: \overleftrightarrow{A B} \Leftrightarrow \overleftarrow{A B}$

Graphically, the cyclic slip of one contact can be observed only when the trajectory hits opposite sides of the slip surface, and cyclic slip of both contacts when opposite corners of the slip surface are hit [cf. Fig. 2(a)].

Table 1 gives an exhaustive list of the possible steady state regimes that may arise under cyclic loading.

\section{BEHAVIOUR MAP}

If the same reasoning is applied but now a simpler set of forcing functions is used, a response mode map (cf. Bree diagram) may be developed for the steady state response of the system, where the axes are the dimensionless load parameters. In order to generate a map including as many as possible of the regimes noted in Table 1, a non-overlapping pulse train of alternating $F_{1}$ and $F_{2}$ is applied [see Fig. 4(a)]. The system is then characterized by only two quantities, namely $F_{1}^{\mathrm{MAX}} /(f P)$ and $F_{2}^{\mathrm{MAX}} /(f P)$. This allows points in $\left[F_{1}^{\mathrm{MAX}} /(f P), F_{2}^{\mathrm{MAX}} /(f P)\right]$ space to be defined that fully encapsulate the loading history. The corresponding trajectories may then be determined in $\left[F_{1} /(f P), F_{2} /(f P)\right]$ space for the loading cycle, taking into account the effect of changing $F_{3}$. The regimes found are depicted in Fig. 4(b) and it is seen that all but one of the possible behaviour patterns outlined in Table 1 (cyclic slip) occur.

\subsection{Shakedown}

It can be seen from Fig. 4(b) that there is a significant region in which the loading history results in frictional shakedown. This prompts a verification of the shakedown limit using the Melan theorem [2]. The shakedown limit may be found by postulating a residual stress, $F_{3}^{*}$, such that under a given cycle defined by $F_{1}^{\mathrm{MAX}}$ and $F_{2}^{\mathrm{MAX}}$ in the form of Fig. 4(a) there is an incipient slip in opposite directions at the extreme points in the cycle. Thus, if

$$
\begin{aligned}
& F_{1}^{\mathrm{MAX}}-F_{3}^{*}=f P(\text { or }-f P) \\
& F_{2}^{\mathrm{MAX}}-F_{3}^{*}=-f P(\text { or }+f P)
\end{aligned}
$$

these two equations may be solved for $F_{3}^{*}$ to give

$$
F_{3}^{*}=\frac{F_{1}^{\mathrm{MAX}}+F_{2}^{\mathrm{MAX}}}{2}
$$

which defines the shakedown limit as the line

$$
F_{1}^{\mathrm{MAX}}-F_{2}^{\mathrm{MAX}}= \pm 2 f P
$$

This clearly demarcates the shakedown region in Fig. 4(b) from the walking regions. It is noted that the lines $F_{1}^{\mathrm{MAX}}= \pm 2 f P$ and $F_{2}^{\mathrm{MAX}}= \pm 2 f P$ represent limit states of full slip. Graphically, however, it may be stated that, in the most general case, if the loading trajectory $F_{1}, F_{2}$ in space never crosses the boundaries of its operating slip surface square in the steady state, then the structure has shaken down.

Table 1 Possible steady state responses under cyclic loading

\begin{tabular}{lll}
\hline Regimes & Notation & Plastic analogy \\
\hline Permanent adhesion & $\hat{A} \hat{B}$ & Elasticity \\
Frictional shakedown & $\vec{A} \hat{B} \Rightarrow \hat{A} \hat{B}$ & Elastic shakedown \\
Gross sliding & $\overrightarrow{A B} \Leftrightarrow \overleftrightarrow{A B}$ & Limit state \\
Walking (rigid body motion) & $\vec{A} \hat{B} \Leftrightarrow \hat{A}$ & Ratcheting (incremental collapse) \\
Cyclic slip & $\vec{A} \overleftrightarrow{\leftrightarrow} \Leftrightarrow \overleftrightarrow{A}$ & Cyclic plasticity (plastic shakedown) \\
& $\vec{A} \hat{B} \Leftrightarrow \overleftrightarrow{A} \hat{B}$ & \\
\hline
\end{tabular}



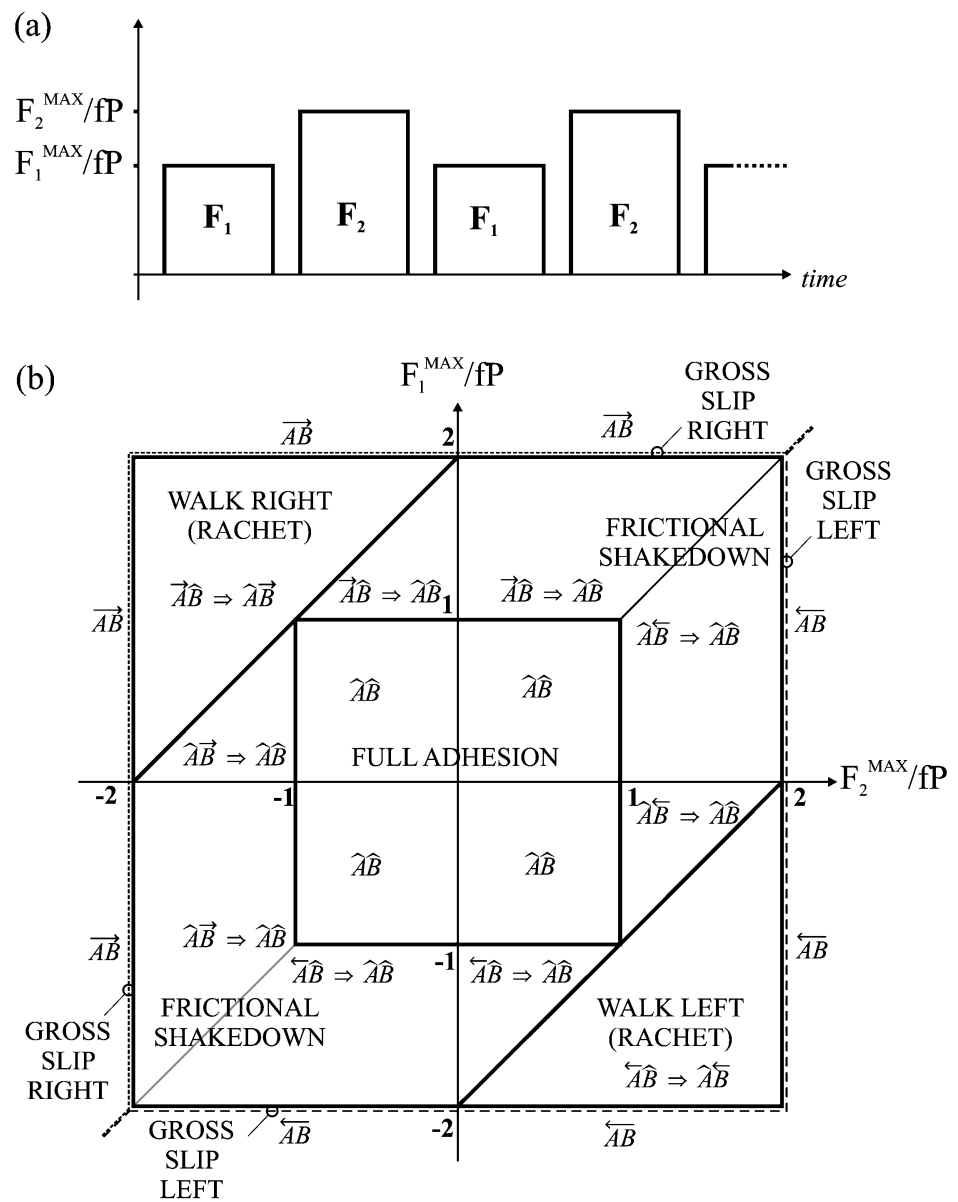

Fig. 4 (a) Alternating pulse train of the driving forces $F_{1}$ and $F_{2}$ of magnitudes $F_{1}^{\mathrm{MAx}} /(f P)$ and $F_{2}^{\mathrm{MAX}} /(f P)$ respectively; (b) Bree type behaviour map with each pulse train representing a point in space in the steady state

This property, where the shakedown limit is only infinitesimally below the limit state, is characteristic of systems with very limited redundancy. The same behaviour is exhibited by an ideally elastoplastic beam in pure bending.

\section{CONCLUSION}

This paper provides the framework for a strong analogy between continuum plasticity and Coulomb frictional contact. It is shown that there is a direct correspondence, under steady state cyclic conditions, between the responses that are possible. This enables the response of the structure to be summarized in a compelling graphical way on a 'map' that corresponds to the celebrated Bree diagram. Also, the shakedown theorems of Melan and Koiter may be employed to infer bounding conditions for the shakedown state.
Although the paper has utilized a very simple structure where the frictional contacts are discrete and of infinitesimal extent, the response may be generalized to configurations in which there is a distributed contact patch, split into regions of partial slip and adhesion, for example.

\section{REFERENCES}

1 Bree, J. Elastic-plastic behavior of thin tubes subjected to high internal pressure and intermittent high heat fluxes with applications to fast-nuclear-reactor fuel elements. J. Strain Analysis, 1967, 2(3), 226-238.

2 Melan, E. Theorie statisch unbestimmter Systeme aus ideal-plastischem Baustoff. Sitzungsber. d. Akad. d. Wiss., Wien, 1936, 2A(145), 195-218.

3 Koiter, W. T. General theorems for elastic-plastic solids. In Progress in solid mechanics, vol. I, 1960, pp. 165-221 (North-Holland Publishing Company). 


\section{APPENDIX}

\section{Notation}

$\hat{A} \quad$ contact $A$ adheres to the bar

$\vec{A} / \overleftarrow{A} \quad$ contact $A$ slips to the right/left relative to the bar

$f \quad$ coefficient of friction

$F_{1} \quad$ tension applied to the left-hand side of the bar
$F_{2} \quad$ tension applied to the right-hand side of the bar

$F_{3} \quad$ internal force in the bar

$k \quad$ bar stiffness

$P \quad$ normal force at contact

$Q \quad$ resultant shear force

$T$ shear force at contact 